\title{
Neumomediastino espontáneo con sospecha de síndrome de Boerhaave
}

\author{
Héctor Marín Ortegaa ${ }^{a}$ Iñaki Martínez Rodríguez ${ }^{\mathrm{b}}$, Patricia Mifsut Porcel ${ }^{\mathrm{a}}$, Leire Agirre Etxabe ${ }^{\mathrm{a}}$
}

\begin{abstract}
a Médico Interno Residente de Cirugía General y del Aparato Digestivo, Hospital Universitario Cruces (Barakaldo)

b Servicio de Cirugía General y del Aparato Digestivo, Hospital Universitario Cruces (Barakaldo)

Correspondencia: Héctor Marín Ortega. Hospital Universitario Cruces, Plaza Cruces s/n 48903, Barakaldo, Bizkaia, España.
\end{abstract}

Correo electrónico: $\underline{h}$ marinortega@hotmail.com

Recibido el 2 de febrero de 2012.

Aceptado para su publicación el 21 de agosto de 2012.

\section{RESUMEN}

El neumomediastino espontáneo es una rara entidad definida como la presencia de aire en el mediastino de causa no yatrógena ni traumática. Suele afectar a pacientes en edad pediátrica, generalmente asociado a la crisis asmática. Otros desencadenantes son el Valsalva, ejercicio intenso, vómitos, cetoacidosis diabética o consumo de drogas. Clínicamente es típica la asociación de dolor torácico y disnea y generalmente se trata de un proceso benigno que se resuelve con tratamiento conservador. Sin embargo, su presentación clínica suele resultar más confusa en pacientes adolescentes y adultos, donde en ocasiones es indistinguible de la ruptura espontánea del esófago (Síndrome de Boerhaave), siendo fundamental un correcto diagnóstico diferencial entre ambas entidades dadas las diferentes implicaciones terapéuticas y pronósticas, como en el caso que presentamos. La presentación clínica puede ser idéntica. No obstante, los pacientes con perforación esofágica tienden a la sepsis progresiva, con mortalidades elevadas si el diagnóstico se retrasa. En nuestro caso el aspecto del paciente, la leucocitosis, el dolor abdominal y la historia compatible nos hizo plantearnos esa posibilidad. Siempre que exista la sospecha de perforación esofágica está indicado practicar más pruebas diagnósticas, de las cuales la TAC con contraste oral hidrosoluble es de elección, pues permite valorar el estado de la pared esofágica y la presencia de colecciones líquidas asociadas.

Palabras Clave. Dolor torácico, Neumomediastino, Enfisema subcutáneo, Perforación esofágica.

\section{ABSTRACT}

Spontaneous Pneumomediastinum, Boerhaave Syndrome suspected.

Spontaneous pneumomediastinum is a rare entity which is defined as the presence of air in the mediastinum not caused by trauma or secondary to any medical procedure. It usually affects patients in the pediatric age, most frequently in the context of an asthma exacerbation. Other triggers are Valsalva maneuvers, intense exercise, vomiting, diabetic ketoacidosis and drug use. It is usually presented as an association of chest pain and dyspnea and it is generally a benign process that resolves with conservative treatment. However, clinical presentation is more confusing in adolescents and adult patients, where it is sometimes indistinguishable from spontaneous rupture of the esophagus (Boerhaave syndrome). A correct differential diagnosis between these two entities is essential, given the different therapeutic and prognostic implications, as in the case we present. Clinical presentation may be identical. However, patients with esophageal perforation tend to progressive sepsis with high mortality rates if diagnosis is delayed. In our case, the patient's appearance, leukocytosis, abdominal pain and a compatible history supported this possibility. It is mandatory to practice more diagnostic tests whenever rupture of the esophagus is suspected. The practice of a CT exploration after the oral administration of water-soluble contrast may be the best choice because it allows to assess the esophageal wall and the presence of associated fluid collections.

Key words: Thoracic Pain, Mediastinal emphysema, Subcutaneous emphysema, Spontaneous perforation of the esophagus.

\section{INTRODUCCIÓN}

El neumomediastino espontáneo es una entidad infrecuente que suele afectar a pacientes en edad pediátrica y adolescentes'. Su presentación clínica suele resultar más confusa en pacientes de mayor edad, donde en ocasiones es indistinguible de la perforación esofágica espontánea (síndrome 
de Boerhaave), siendo fundamental un correcto diagnóstico diferencial dadas las diferentes implicaciones pronósticas entre ambas entidades.

\section{OBSERVACIONES CLÍNICAS}

Presentamos el caso de un varón de 20 años de edad, sin antecedentes de interés. Acude a urgencias tras 4 días de molestias abdominales y vómitos. Inquieto y alterado, sudoroso. Tensión arterial 167/67 mmHg, taquicárdico. Afebril. A la exploración destaca dolor abdominal y ligera defensa en epigastrio e hipocondrio derecho. Leucocitosis de $19.200 / \mathrm{mm} 3$. Durante su estancia en urgencias, el paciente presenta súbito empeoramiento de su estado tras nuevo episodio de vómitos, refiriendo dolor centrotorácico seguido de enfisema subcutáneo supraclavicular y cervical. La radiografía de tórax (fig. 1) muestra un importante enfisema subcutáneo en partes blandas supraclaviculares y axilares. No se aprecian neumotórax ni derrame pleural asociados.

Ante la sospecha de rotura espontánea de esófago se solicita TAC toracoabdominal con contraste oral hidrosoluble (fig. 2) que identifica un importante enfisema de tejido celular subcutáneo que se distribuye por la totalidad de espacios cervicales, parafaríngeos, paravertebrales, espacio epidural de canal medular y mediastino. No se identifican colecciones mediastínicas ni fugas del contraste oral. Dado el contexto, el estado clínico del paciente y los hallazgos analíticos se decide ingreso con tratamiento conservador con el posible diagnóstico de perforación espontánea de esófago.

Durante las primeras 24 horas de ingreso el paciente persiste con vómitos, dolor torácico, que ha aumentado de intensidad, y leucocitosis (20.200/mm3), por lo que se decide repetir la TAC con contraste oral que revela hallazgos similares a la exploración previa, con ausencia de fugas del contraste. La evolución posterior es favorable, con disminución progresiva del enfisema subcutáneo, normalización analítica y mejoría clínica, siendo dado de alta al $5^{\circ}$ día.

\section{COMENTARIOS}

El neumomediastino espontáneo se define como la presencia de aire en el mediastino de causa no yatrógena ni traumática. La incidencia descrita en la literatura oscila entre 1:800 y 1:42.000 pacientes atendidos en urgencias ${ }^{2}$. El evento desencadenante es una fuga aérea en el espacio alveolar; posteriormente el aire diseca la vaina broncovascular de manera centrípeta, hasta alcanzar el mediastino debido a la presión negativa relativa entre ambos compartimentos ${ }^{2}$. Excepcionalmente, el origen de la fuga aérea es la vía aérea extrapulmonar. Generalmente la distensibilidad de las partes blandas mediastínicas y cervicales impide el incremento de presión intratorácica, aunque se han descrito casos excepcionales de neumomediastino con compromiso hemodinámico, sobre todo en casos asociados a barotrauma, entidad conocida como neumomediastino a tensión ${ }^{3}$.

El desencadenante más frecuente es la crisis asmática ${ }^{4}$. Se calcula que en mayor o menor grado acompaña a hasta un $5 \%$ de las crisis asmáticas en edad infantil. En adultos esta relación es menos frecuente. Otros factores implicados son el Valsalva, ejercicio intenso, vómitos, cetoacidosis diabética, cuerpos extraños o consumo de drogas (cocaína, éxtasis), entre otros ${ }^{2,3,5}$.

La clínica típica es la asociación de dolor torácico y disnea. El dolor torácico es el síntoma más frecuente 1 . Es retroesternal, opresivo, de instauración brusca, que se incrementa con la inspiración profunda e irradiado a hombros. Síntomas menos constantes son: disfagia, dolor cervical, disfonía y dolor abdominal ${ }^{4}$.

Los hallazgos exploratorios pueden ser característicos, pero un tercio de los pacientes tienen una exploración normal. El enfisema subcutáneo es uno de los hallazgos clásicamente descritos, y probablemente se trate del hallazgo más sensible, aunque es poco específico por si mismo. El signo de Hamman, crepitación sincrónica con el latido cardiaco a la auscultación, es también muy sugestivo, aunque relativamente poco frecuente ${ }^{6}$.

La radiografía de tórax permite confirmar el diagnóstico. En hasta la mitad de los casos los signos radiográficos pueden ser sutiles y se requiere la proyección lateral para llegar al diagnóstico. Los signos radiográficos suelen consistir en la aparición de gas retroesternal y delimitando estructuras como la aorta descendente, el timo en los niños (signo de la aleta tímica, signo del spinnaker) o la base cardiaca respecto al diafragma izquierdo (signo del diafragma continuo) ${ }^{7}$.

El diagnóstico diferencial es amplio, debiéndose diferenciar la entidad del resto de causas de dolor torácico. No obstante, es importante por 
sus implicaciones terapéuticas y pronósticas realizar un correcto diagnóstico diferencial con la ruptura espontánea del esófago, sobre todo en pacientes adultos con historia sugestiva y apariencia de gravedad $^{8}$. La presentación clínica puede ser indistinguible. No obstante, los pacientes con perforación esofágica tienden a la sepsis progresiva, con mortalidades elevadas si el diagnóstico se retrasa. En nuestro caso, el aspecto del paciente, la leucocitosis, el dolor abdominal y la historia compatible nos hizo plantearnos esa posibilidad. En estos casos está indicado practicar más pruebas diagnósticas, de las cuales la TAC es de elección, pues permite valorar el estado de la pared esofágica y la presencia de colecciones líquidas asociadas ${ }^{9}$. La sensibilidad de la técnica se incrementa con el empleo de contraste oral, preferiblemente hidrosoluble.

El tratamiento consiste en el reposo, analgesia y evitación de maniobras de Valsalva ${ }^{5}$, acompañado del tratamiento del proceso asociado si lo requiriese. La oxigenoterapia con concentraciones elevadas puede acelerar la reabsorción del aire, pero no está indicada de manera sistemática.

La resolución suele darse en 3 a 5 días. La recurrencia es rara, inferior en todo caso al $5 \%{ }^{1}$. Dado el carácter benigno de la entidad, no se recomienda ningún seguimiento especial.

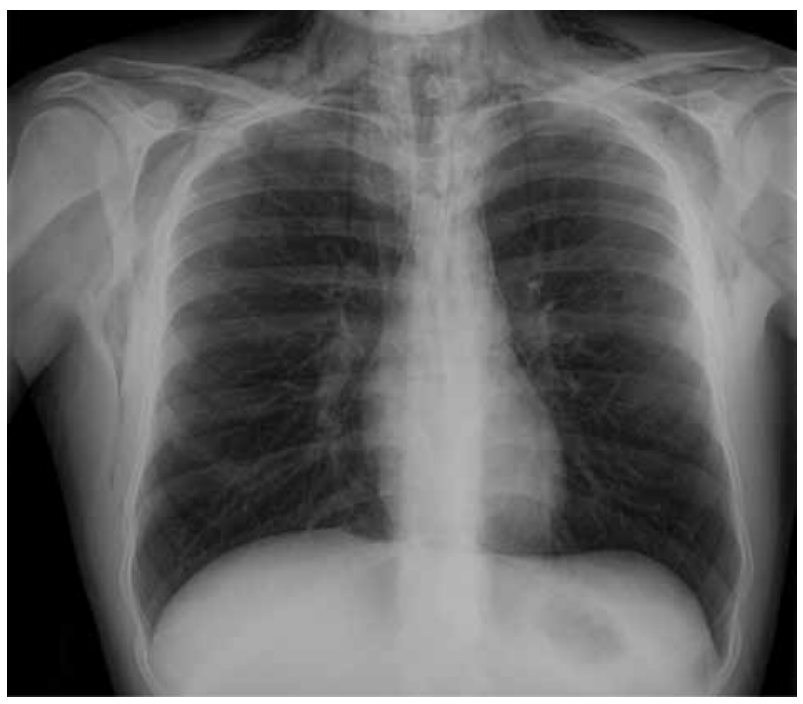

Figura 1. Radiografía de tórax anteroposterior. Enfisema subcutáneo supraclavicular y en espacios axilares, en localización paratraqueal bilateral e infracardíaco.

\section{BIBLIOGRAFÍA}

1. Dekel B, Paret G, Szeinberg A, Vardi A, Barzilay Z. Spontaneous pneumomediastinum in children: clinical and natural history. Eur J Pediatr. 1996; 155(8):695-7.

2. Weissberg D, Weissberg D. Spontaneous mediastinal emphysema. Eur J Cardiothorac Surg. 2004;26(5):885-888.

3. Newcomb AE, Clarke CP. Spontaneous pneumomediastinum: a benign curiosity or a significant problem? Chest. 2005;128:3298-3302.

4. Chiu CY, Wong KS, Yao TC, Huang JL. Asthmatic versus non-asthmatic spontaneous pneumomediastinum in children. Asian Pac J Allergy Immunol. 2005;23(1):19-22.

5. Macia I, Moya J, Ramos R, Morera R, Escobar I, Saumench $\mathrm{J}$ et al. Spontaneous pneumomediastinum: 41 cases. Eur $\mathrm{J}$ Cardiothorac Surg. 2007;31(6):1110-4.

6. Campillo-Soto A, Coll-Salinas A, Soria-Aledo V, BlancoBarrio A, Flores-Pastor B, Candel-Arenas $M$ et al. Neumomediastino espontáneo: estudio descriptivo de nuestra experiencia basada en 36 casos. Arch Bronconeumol. 2005;41(9):528-531.

7. Gurney JW, Winer-Muram HT. Neumomediastino. En: Gurney JW, ed. Los 100 diagnósticos principales en tórax. Madrid: Elsevier España; 2004.p.166-168.

8. Forshaw MJ, Khan AZ, Strauss DC, Botha AJ, Mason RC. Vomiting-induced pneumomediastinum and subcutaneous emphysema does not always indicate Boerhaave's syndrome: report of six cases. Surg Today. 2007;37(10):888-92.

9. De Lutio di Castelguidone E, Pinto A, Merola S, Stavolo C, Romano L. Role of Spiral and Multislice Computed Tomography in the evaluation of traumatic and spontaneous oesophageal perforation. Our experience. Radiol Med. 2005;109(3):252-9.

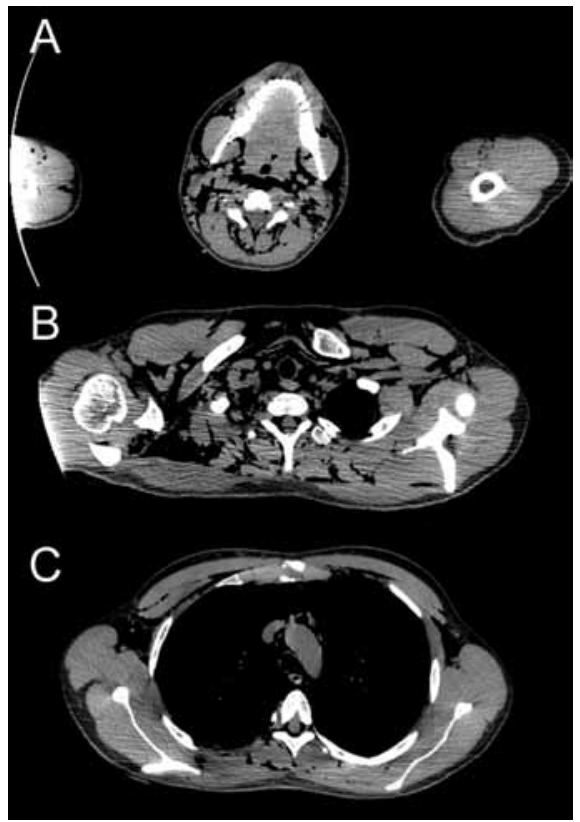

Figura 2 - A) TAC cervical. Importante enfisema subcutáneo en espacios cervicales, parafaríngeos, paravertebrales y espacio epidural de canal medular. B) TAC cervical. Corte axial a nivel de vértices pulmonares. Importante enfisema subcutáneo en espacios axilares bilaterales y pared torácica anterior. Neumomediastino. C) TAC torácico. Corte axial a nivel de cayado aórtico. Enfisema subcutáneo en pared torácica anterior, musculatura paravertebral y espacio epidural de canal medular. Neumomediastino. Ausencia de colecciones mediastínicas y de fugas de contraste a nivel esofágico. 